Proceedings of the 2011 Winter Simulation Conference

S. Jain, R. R. Creasey, J. Himmelspach, K. P. White, and M. Fu, eds.

\title{
OPTIMIZING LOCAL PICKUP AND DELIVERY WITH UNCERTAIN LOADS
}

\author{
Weiwei Chen \\ GE Global Research \\ Management Sciences Lab \\ 1 Research Circle \\ Niskayuna, NY 12309, USA
}

\author{
Jie Song \\ Peking University \\ Industrial Engineering and Management \\ 298 Chengfu Road \\ Beijing 100871, CHINA
}

\author{
Leyuan Shi \\ University of Wisconsin-Madison \\ Industrial and Systems Engineering \\ 1513 University Avenue \\ Madison, WI 53706, USA
}

\begin{abstract}
The local pickup and delivery problem (LPDP) is an essential operational problem in intermodal industry. While the problem with deterministic settings is already difficult to solve, in reality, there exist a set of loads, called uncertain loads, which are unknown at the beginning of the day. But customers may call in during the day to materialize these loads. In this paper, we call the LPDP considering these uncertain loads as the stochastic LPDP. The problem description and the mathematical modeling of stochastic LPDP are discussed. Then, a simulation-based optimization approach is proposed to solve the problem, which features in a fast solution generation procedure and an intelligent simulation budget allocation framework. The numerical examples show the best strategy to consider the stochastic loads in the planning process and validate the benefits compared to its deterministic counterpart.
\end{abstract}

\section{INTRODUCTION}

In recent years, competition in the transportation and logistics sector has increasingly intensified. The efforts to maintain viable profit margins are further complicated by rising personnel and fuel costs. As a result, the local pickup and delivery problem (LPDP) has drawn a great deal of interest in intermodal transportation. Truck/rail intermodal transportation combines the cost-effectiveness of rail with the flexibility of trucks. When freight is transported, it is first loaded onto a trailer or a container which is moved from the shipper to a nearby rail ramp by truck. Then the freight is conveyed by rail to a ramp close to its destination. Finally it is delivered to the consignee by truck. The truck portion of intermodal transportation is called drayage, and can be modeled as a LPDP. Although the truck portion is much shorter than the rail portion, its cost is a significant component of the total cost, due to the growth in service demand and higher labor costs compared to rail delivery (Morlok and Spasovic 1994).

The LPDP is concerned with the optimal movement of a set of loads between customer locations and rail ramps in a local service area over a relatively short planning horizon. The basic operations involved in a LPDP can be described as follows (Wang and Regan 2002; Pi, Pan, and Shi 2008): At the beginning of each work day, a fixed number of vehicles are positioned throughout the service area. A vehicle can serve only one load at a time. After the delivery of a load, it runs for another load immediately or becomes idle. Served loads generate revenues and unserved ones may be subcontracted to other carriers (for an 
additional cost) or simply lost (without generating any revenue). Empty movements of vehicles incur costs. The optimization objective is to maximize the overall profit over the planning horizon. To achieve this objective, the planners must serve as many loads as possible and minimize the empty movements.

In the literature, extensive research has been done in solving static LPDPs, which means at the time of planning, all inputs (such as loads, drivers, traveling time) are deterministic and known in advance (Savelsbergh and Sol 1995). But in the real world, the system evolves dynamically due to refreshed data or uncertain information. For instance, a traffic delay may cause the driver's remaining schedule to be non-executable. Therefore, the solution approach for a static LPDP is usually applied in a dynamic manner to handle new information and make adjusted decisions (Berbeglia, Cordeau, and Laporte 2010). The planning is made at given time, such as every hour, or when an event is triggered, such as when a new load is confirmed or a driver is delayed due to bad traffic. Dynamic solution scheme is deployed to adapt to the highly dynamic characteristics and uncertainties of LPDPs, and has been proven to be more flexible and efficient than solving a single static LPDP (Ileri 2006).

The above mentioned static and dynamic version of LPDPs only consider the deterministic information, which has been confirmed at the time of planning. However, in real operations, uncertainty raises an issue to the planners. One stochastic input to the planning is the travel time between two locations in the local network. With modern communication technique and applying the LPDP solvers in a dynamic manner, the impact of uncertain travel time can be greatly alleviated. Another uncertain resource is the loads (also known as tasks for drivers). There exist certain amount of uncertain loads, called stochastic loads, which may arise unexpectedly during the operating period (Erera and Smilowitz 2008). In some markets, the proportion of stochastic loads can be considerable, up to $30-40 \%$ of the daily loads. The implementation of dynamic LPDP solution approaches can response to these unexpected loads by re-optimizing the routes. However, if we consider the stochastic loads in advance by constructing routes that are close to those stochastic loads, a driver might be nearby at the time when a load is called in and can be immediately sent to serve it. As a result, the empty movement distance is shorten, and the overall dispatch capacity is potentially increased. In this paper, we study the benefit and solution approach of taking into account stochastic loads in the planning process, and we call this problem a stochastic LPDP.

The rest of this paper is organized as follows. In Section 2, the deterministic LPDP is briefly reviewed, and the modeling of stochastic LPDP is presented. Then, a simulation-based optimization approach is proposed to solve stochastic LPDP in Section 3. The experimental results are shown in Section 4, and conclusion is made in Section 5.

\section{STOCHASTIC LPDP MODEL}

\subsection{Deterministic LPDP Model and Solution Approaches}

Before discussing stochastic LPDPs, we briefly introduce the deterministic (static) LPDPs. It can be modeled as a mixed integer program (Wang and Regan 2002; Pi, Pan, and Shi 2008) as follows.

Sets:

- $K=\{1, \ldots,|K|\}$ : the set of drivers.

- $L=\{1, \ldots,|L|\}$ : the set of nodes representing loads.

- $S=\{|L|+1, \ldots,|L|+|K|\}$ : the set of start nodes, as the start location of drivers.

- $H=\{|L|+|K|+1, \ldots,|L|+2|K|\}$ : the set of end nodes, as the home location of drivers.

Parameters:

- $T_{i j}, i \in L \cup S, j \in L \cup H$ : the time needed to serve load $i$ and then travel to the origin of load $j$.

- $a_{i}, i \in L \cup S$ : the earliest start time of each node. For $i \in S, a_{i}$ means the initial available time of driver $i-|L|$. 


\section{Chen, Song, and Shi}

- $b_{i}, i \in L \cup H$ : the latest start time of each node. For $i \in H, b_{i}$ means the latest time when driver $(i-|L|-|K|)$ must return to home location. $\forall i \in L,\left[a_{i}, b_{i}\right]$ denotes the time window of load $i$.

- $W_{i}, i \in L \cup S$ : the revenue by serving load $i . \forall i \in S, W_{i}=0$.

- $C_{i j}, i \in L \cup S, j \in L \cup H$ : the cost of traveling from the origin of load $i$ to the origin of load $j$.

\section{Variables:}

- $\quad x_{k i j}, k \in K, i \in L \cup S, j \in L \cup H$ : binary variables. $x_{k i j}=1$ if driver $k$ serves load $j$ followed by load $j ; x_{k i j}=0$ otherwise. Note that for any $k \in K, x_{k i j}$ is fixed to zero for $i \in S \backslash\{k+|K|\}$ or $j \in H \backslash\{k+|L|+|K|\}$.

- $t_{i}, i \in L \cup S \cup H$ : service start time of each node.

Now we give a formulation based on the multi-commodity network flow as follows. Objective:

$$
\max z=\sum_{i \in L \cup S} W_{i} \cdot \sum_{j \in L \cup H} \sum_{k \in K} x_{k i j}-\sum_{i \in L \cup S} \sum_{j \in L \cup H} C_{i j} \cdot \sum_{k \in K} x_{k i j}
$$

Subject to:

$$
\begin{gathered}
\sum_{j \in L \cup H} \sum_{k \in K} x_{k i j}=1 \quad \forall i \in S, \\
\sum_{i \in L \cup S} \sum_{k \in K} x_{k i j}=1 \quad \forall j \in H, \\
\sum_{i \in L \cup S} x_{k i j}=\sum_{l \in L \cup H} x_{k j l} \quad \forall k \in K, j \in L, \\
\sum_{i \in L \cup S} \sum_{k \in K} x_{k i j} \leq 1 \quad \forall j \in L, \\
t_{i}+T_{i j}-t_{j} \leq\left(1-\sum_{k \in K} x_{k i j}\right)\left(b_{i}+T_{i j}-a_{j}\right) \quad \forall i \in L \cup S, j \in L \cup H, \\
t_{i} \geq a_{i} \quad \forall i \in L \cup S, \\
t_{i} \leq b_{i} \quad \forall i \in L \cup H .
\end{gathered}
$$

Here, (1) represents the revenues of served loads minus the traveling costs. Constraints (2) (3) (4) are standard multi-commodity network flow constraints. Constraints (5) require each load to be served no more than once. Constraints (6) are the temporal relations between consecutive nodes. Constraints (7) and (8) are the load time window constraints.

The LPDP is difficult to solve, and has been proven to be NP-hard (Erera and Smilowitz 2008). Some researches tried to solve specific instances to optimally using mathematical programming (Arunapuram, Mathur, and Solow 2003; Lu and Dessouky 2004). Due to the large scale of the problem, fast and good heuristic methods are popular choices in solving these problems. In Wang and Regan (2002), an iterative solution approach is proposed to deal with hard time window constraints. An over-constrained and a under-constrained problem are solved at each iteration to guide the search. In Powell, Shapiro, and Simao (2002), a multi-stage dynamic program model is introduced, and adaptive dynamic programming techniques are used to obtain high-quality solutions. Pi, Pan, and Shi (2008) propose a hybrid nested partitions and mathematical programming (HNP-MP) approach which can provide good solutions to large-scale LPDPs efficiently. Other useful heuristic methods can be found in Powell and Carvalho (1998); Xu et al. (2001); Campbell and Savelsbergh (2004); Funke, Grunert, and Irnich (2005). 


\subsection{Modeling of Stochastic LPDP}

In Section 1, the benefits of considering stochastic loads in the planning are introduced. A typical scenario of stochastic LPDP is shown in Fig. 1, where one driver is serving three deterministic loads and two stochastic loads. In order to model the stochastic LPDP, there are two key factors to be discussed: 1) how to define the probability of a stochastic load, and 2) what difference in a schedule will be observed if stochastic loads are considered.

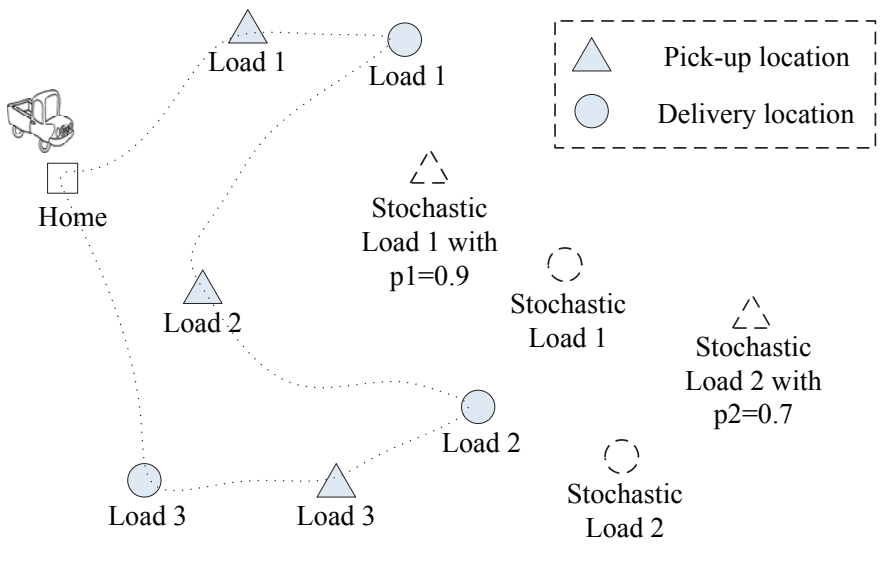

Figure 1: A stochastic LPDP example.

We first extend the set definitions in Section 2.1 for the stochastic LPDP as follows.

- $L_{D}=\left\{1,2, \ldots,\left|L_{D}\right|\right\}$ : the set of deterministic loads, which are known before the planning starts.

- $L_{S}=\left\{\left|L_{D}\right|+1,\left|L_{D}\right|+2, \ldots,\left|L_{D}\right|+\left|L_{S}\right|\right\}$ : the set of stochastic loads. Load $i\left(i \in L_{S}\right)$ may happen with probability $p_{i}$ during the planning period.

- $L=L_{D} \cup L_{S}$ : the set of all possible loads.

\subsubsection{Occurrence Probability of Stochastic Loads}

In the dynamic solution scheme, we divide the time horizon into $T(T>0)$ stages, and at the beginning of each stage, all the information is refreshed and the schedule is re-optimized. The occurrence probability of a stochastic load may vary at different stages. The probability distribution of load $i\left(i \in L_{S}\right)$ can be described as $p_{i}(t), t \in\{1, \ldots, T\}$. An example is shown in Fig. 2. Within each stage (e.g., 6:00 - 7:00), the occurrence probability is constant. Since all information is refreshed at the beginning of each stage, we drop the $t$ in $p_{i}(t)$ in the following context.

We have the following assumption for simplicity. Assumption 1 usually holds in reality: each stochastic load can represent a retailer, such as Walmart and Target, and thus their needs to initiate a load are independent.

Assumption 1 The probability distribution of each stochastic load is independent with each other.

Prior information of experienced dispatch expert or historical data can be used to estimate the occurrence probability during each period. One of the simplest ways to estimate the probability is to calculate the frequency of this load's appearance during the same time period in historical data. We define this probability as a parameter as follows.

- $\quad p_{i}, i \in L$ : the probability that load $i$ will happen. For $i \in L_{D}$, we always have $p_{i}=1$. For $i \in L_{S}$, $0<p_{i}<1$. 
Chen, Song, and Shi

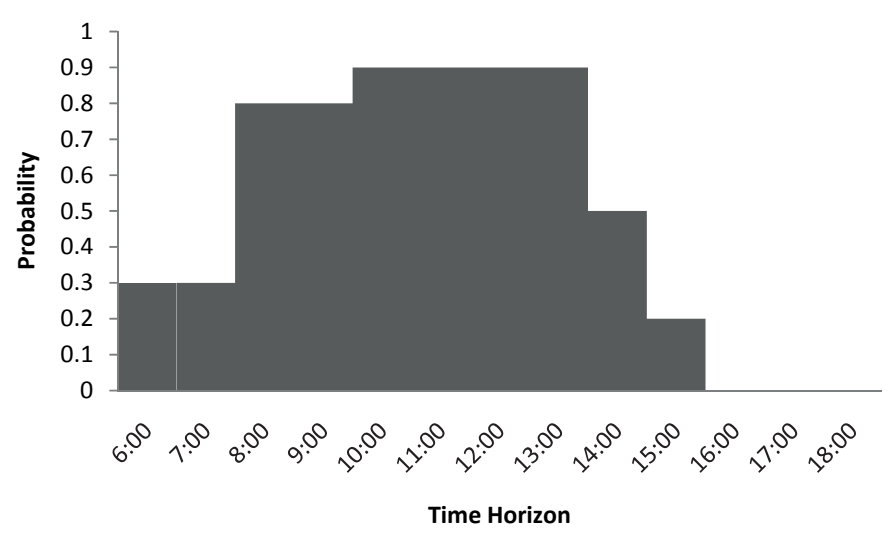

Figure 2: Probability distribution of a stochastic load.

\subsubsection{Scheduling with Stochastic Loads}

We first denote a schedule $\pi(\pi \in \Pi)$ as a solution to a LPDP. Each schedule $\pi$ consists of a route for each driver. Denote $(i, j)$ as a schedule component where load $j$ is served followed by load $i$ by the same driver. Given a schedule with component $(i, j)$ in which $i$ is a stochastic load, there are two possible scenarios when a driver is sent to the origin of load $i$ : 1) If load $i$ has been confirmed before the driver arrives, serve load $i$ and go to the origin of the next load $j$;2) If load $i$ has not been confirmed when the driver arrives, go to the origin of load $j$ directly. This process is described in Fig. 3.

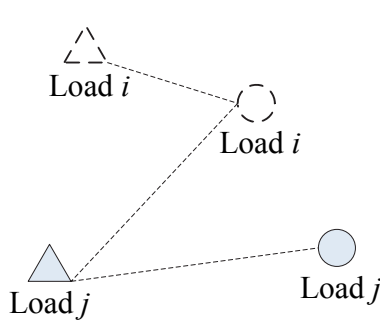

(1) Load $i$ is confirmed

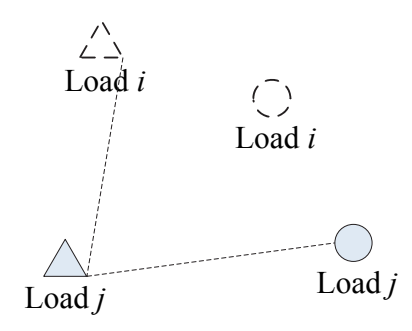

(2) Load $i$ is not confirmed

Figure 3: Two possible scenarios for serving a stochastic load $i$.

We further extend the parameters for stochastic LPDP.

- $C_{i j}, i \in L \cup S, j \in L \cup H$ : the total traveling cost from the original of load $i$ to the destination of load $i$, and then to the origin of load $j$. It represents the cost of scenario 1 in Figure 3 .

- $C_{i j}^{\prime}, i \in L_{S} \cup S, j \in L \cup H$ : the traveling cost from the origin of load $i$ to the origin of load $j$. It represents the cost of scenario 2 in Figure 3. For $i \in S, j \in L_{D} \cup H$, we always have $C_{i j}=C_{i j}^{\prime}$.

- $T_{i j}, i \in L \cup S, j \in L \cup H$ : the time needed to serve load $i$ and then travel to the origin of load $j$, if load $i$ materializes.

- $T_{i j}^{\prime}, i \in L_{S} \cup S, j \in L \cup H$ : the time needed to travel from the origin of load $i$ to the origin of load $j$, if load $i$ does not materialize.

Therefore, the profit of component $(i, j)$ is a random variable, denoted by $z^{(i, j)} \cdot z^{(i, j)}$ can take two values: 1) If load $i$ materializes, we get revenue $W_{i}$ and incur a cost $C_{i j}$, and thus $z^{(i, j)}=W_{i}-C_{i j}$ with probability $p_{i}$; 2) If load $i$ does not materialize, we get zero revenue and incur a $\operatorname{cost} C_{i j}^{\prime}$, and thus $z^{(i, j)}=-C_{i j}^{\prime}$ with 
Chen, Song, and Shi

probability $\left(1-p_{i}\right)$. The expected profit of component $(i, j)$ can be written as

$$
E\left(z^{(i, j)}\right)=\left(W_{i}-C_{i j}\right) \cdot p_{i}-C_{i j}^{\prime} \cdot\left(1-p_{i}\right)=\left(W_{i}-C_{i j}+C_{i, j}^{\prime}\right) \cdot p_{i}-C_{i, j}^{\prime}
$$

\subsubsection{Mathematical Model of Stochastic LPDP}

In a deterministic LPDP, we always seek a best solution in terms of the total profit. When stochastic loads are considered, there can be a set of possible outcomes given a schedule $\pi$. Naturally, people look for a schedule that has better average performance over possible outcomes, and thus the objective is to find the optimal schedule which maximizes the total expected profit. Under Assumption 1, variable $z^{(i, j)}$ 's are independent. Therefore, for a schedule $\pi \in \Pi$ with multiple components involved, the expected profit of $\pi$ are the linear combination of the expected profit of all components. The mathematical form is expressed as:

$$
\begin{aligned}
E\left(z^{\pi}\right) & =\sum_{(i, j) \in \pi, i \in L \cup S, j \in L \cup H} E\left(z^{(i, j)}\right) \\
& =\sum_{(i, j) \in \pi, i \in L \cup S, j \in L \cup H}\left(\left(W_{i}-C_{i j}+C_{i j}^{\prime}\right) \cdot p_{i}-C_{i j}^{\prime}\right) .
\end{aligned}
$$

In model (1) - (8), a schedule $\pi$ is represented by binary variables $x_{k i j}$. We have extended some definitions of sets and parameters in the previous sections, and the remaining notations can be extended straightforwardly. From (10), the objective function can be written using the new notations as:

$$
\begin{aligned}
E(z) & =\sum_{i \in L \cup S} \sum_{j \in L \cup H}\left[\left(W_{i}-C_{i j}+C_{i j}^{\prime}\right) p_{i}-C_{i j}^{\prime}\right] \cdot \sum_{k \in K} x_{k i j} \\
& =\sum_{i \in L \cup S} p_{i} W_{i} \cdot \sum_{j \in L \cup H} \sum_{k \in K} x_{k i j}-\sum_{i \in L \cup S} \sum_{j \in L \cup H}\left[p_{i} C_{i j}+\left(1-p_{i}\right) C_{i j}^{\prime}\right] \sum_{k \in K} x_{k i j} .
\end{aligned}
$$

We further define:

- $\forall i \in L, \hat{W}_{i}=p_{i} W_{i}$

- $\forall i \in L \cup S, j \in L \cup H, \hat{C}_{i j}=p_{i} C_{i j}+\left(1-p_{i}\right) C_{i j}^{\prime}$.

Then, the objective can be re-written as:

$$
\max E(z)=\sum_{i \in L \cup S} \hat{W}_{i} \cdot \sum_{j \in L \cup H} \sum_{k \in K} x_{k i j}-\sum_{i \in L \cup S} \sum_{j \in L \cup H} \hat{C}_{i j} \cdot \sum_{k \in K} x_{k i j}
$$

Constraints (2) - (8) remain valid for the stochastic case with the extended notations, except constraint (6). Corresponding to Figure $3, T_{i j}$ in constraint (6) can be $T_{i j}$ for scenario 1 , or $T_{i j}^{\prime}$ for scenario 2 . If $T_{i j}^{\prime}=T_{i j}$ for all $i \in L_{S}$, then we can take all stochastic loads into consideration, and solve the stochastic LPDP ((2) (8), (11)) using any solution approach for the deterministic LPDP. However, $T_{i j}$ is usually greater than $T_{i j}^{\prime}$, because $T_{i j}$ also includes traveling time from the origin of load $i$ to the destination of load $i$, the loading time at the origin of $i$ and unloading time at the destination of $i$. Therefore, the overall dispatch capacity is reduced when assuming $T_{i j}^{\prime}=T_{i j}$ and taking more stochastic loads into account. Although the solution obtained is a lower bound of the optimum, it provides a fast approach to generate feasible solutions. In the next section, we propose a simulation-based optimization approach for solving stochastic LPDP, utilizing this fast solution generation approach.

\section{SIMULATION-BASED OPTIMIZATION APPROACH}

Since a schedule will be different whether or not a stochastic load materializes and it is difficult to account for this factor in the constraints, we consider using discrete event simulation to estimate the expected profit. The procedure for each simulation replication is described as follows. 
Chen, Song, and Shi

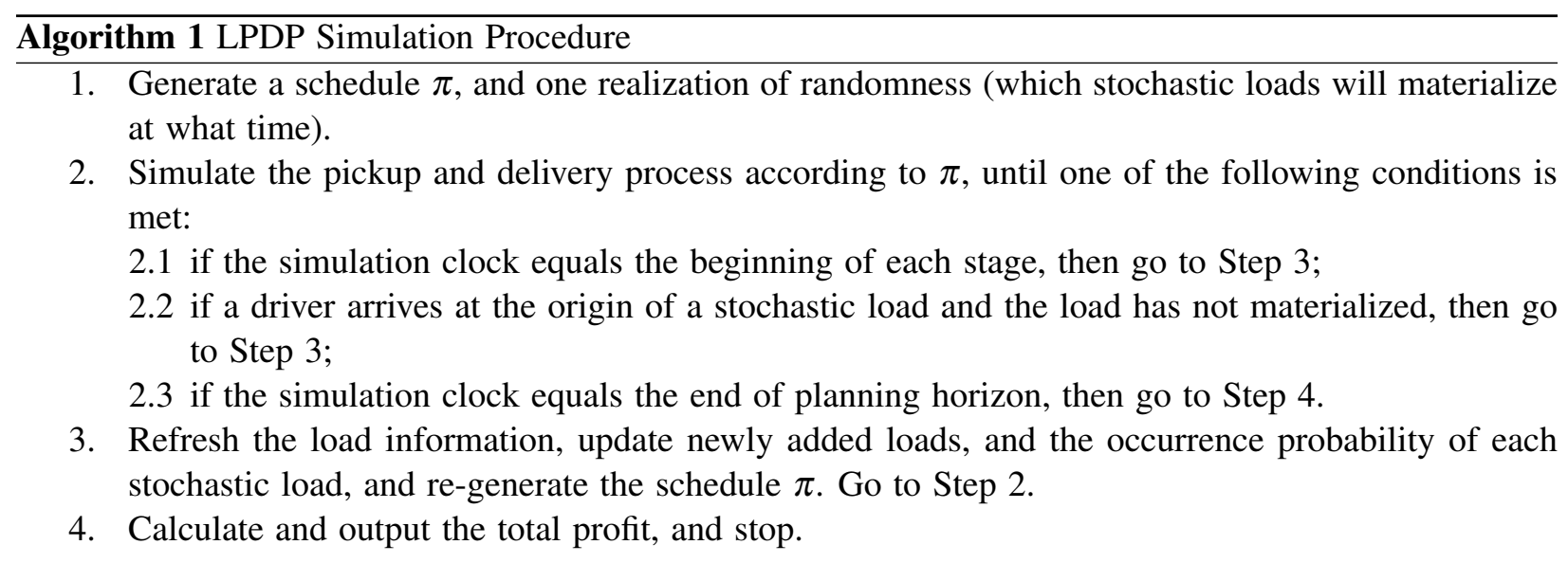

If we are able to simulate a sufficiently large number of replications for each schedule to obtain the mean profit, and evaluate all the available schedules, we will obtain the optimal schedule by simply comparing the mean profits for all schedules. However, this is generally impossible and we need to find an efficient way to optimize the simulation-based system.

\subsection{Policy Optimization}

The solution space for stochastic LPDP is so large that it is almost impossible to compare all the solutions. However, in Section 2.2.3, we know that if we assume $T_{i j}^{\prime}=T_{i j}$, a solution can be generated by solving model ((2) - (8), (11)). We also know that the solution obtained by solving the above model is not optimal for stochastic LPDP, since approximating $T_{i j}^{\prime}$ by $T_{i j}$ lowers the overall dispatch capacity and may reduce the total number of loads served. But this approach at least provides us a feasible solution for stochastic LPDP, whose quality can then be evaluated via simulation. The more stochastic loads we consider, the more empty traveling cost we can save, but the more the capacity we may lose. Therefore, we convert the original problem of finding an optimal schedule to the following new problem:

Problem 1 Given that the solution of a stochastic LPDP with a set of stochastic loads can be obtained by solving model ((2) - (8), (11)), what is the optimal set of stochastic loads that generates the highest expected profit?

Or even a simpler problem:

Problem 2 Given that the solution of a stochastic LPDP with a set of stochastic loads can be obtained by solving model $((2)-(8),(11))$, what is the optimal value of $\bar{p}$, such that considering all the stochastic loads with occurrence probability higher than $\bar{p}$ will generate the highest expected profit?

To reduce the solution space, it is nature to discretize the space of $\bar{p}$. Thus, we end up with comparing the performance of a small number of threshold values, say $\bar{p} \in\{0.5,0.6,0.7,0.8,0.9,1\}$. When $p_{i}$ is too small, it is possible that $E\left(z^{(i, j)}\right)<0$ in (9), and thus load $i$ will never be included in the route when solving model ((2) - (8), (11)). Therefore, a small value of $\bar{p}$ does not need to be considered.

For each value of $\bar{p}$, there is a corresponding set of stochastic loads. So, the schedule $\pi$ in the Algorithm 1 Step 2 and Step 3 can be easily generated by solving the model ((2) - (8), (11)) considering all stochastic loads with $p_{i} \geq \bar{p}$.

\subsection{Simulation Budget Allocation}

In Section 3.1, we convert the original problem into a new problem with a small number of solutions. To find the best solution, we need to simulate each solution for a reasonable number of replications, which is usually time consuming. We apply an efficient approach for simulation optimization, called Optimal 
Computing Budget Allocation (OCBA) (Chen et al. 2000; Chen and Lee 2010). OCBA is a methodology for allocating simulation budget such that the probability of correct selection, the probability that the found solution is actually the best solution, is maximized given the simulation budget. It can be stated as the following theorem, and we refer to Chen and Lee (2010) for details.

Theorem 1 Given a total number of simulation samples $T$ to be allocated to $k$ competing solutions whose performance is depicted by random variables with means $J_{1}, J_{2}, \ldots, J_{k}$, and finite variance $s_{1}^{2}, s_{2}^{2}, \ldots, s_{k}^{2}$ respectively, as $T \rightarrow \infty$, the Approximate Probability of Correct Selection (APCS) can be asymptotically maximized when

$$
\begin{gathered}
\frac{N_{i}}{N_{j}}=\left(\frac{s_{i} / \delta_{b, i}}{s_{j} / \delta_{b, j}}\right)^{2}, i, j \in\{1,2, \ldots, k\}, i \neq j \neq b, \\
N_{b}=s_{b} \sqrt{\sum_{i=1, i \neq b}^{k} \frac{N_{i}^{2}}{s_{i}^{2}}},
\end{gathered}
$$

where $N_{i}$ is the number of simulation replications allocated to solution $i, \delta_{b, i}=\bar{J}_{b}-\bar{J}_{i}$, and $b=\arg \min _{i \in\{1,2, \ldots, k\}} \bar{J}_{i}$.

Using Theorem 1, we have the following Algorithm 2 for solving stochastic LPDP. This sequential algorithm generally allow us to find the best value of $\bar{p}$ more efficiently using the limited computational resources.

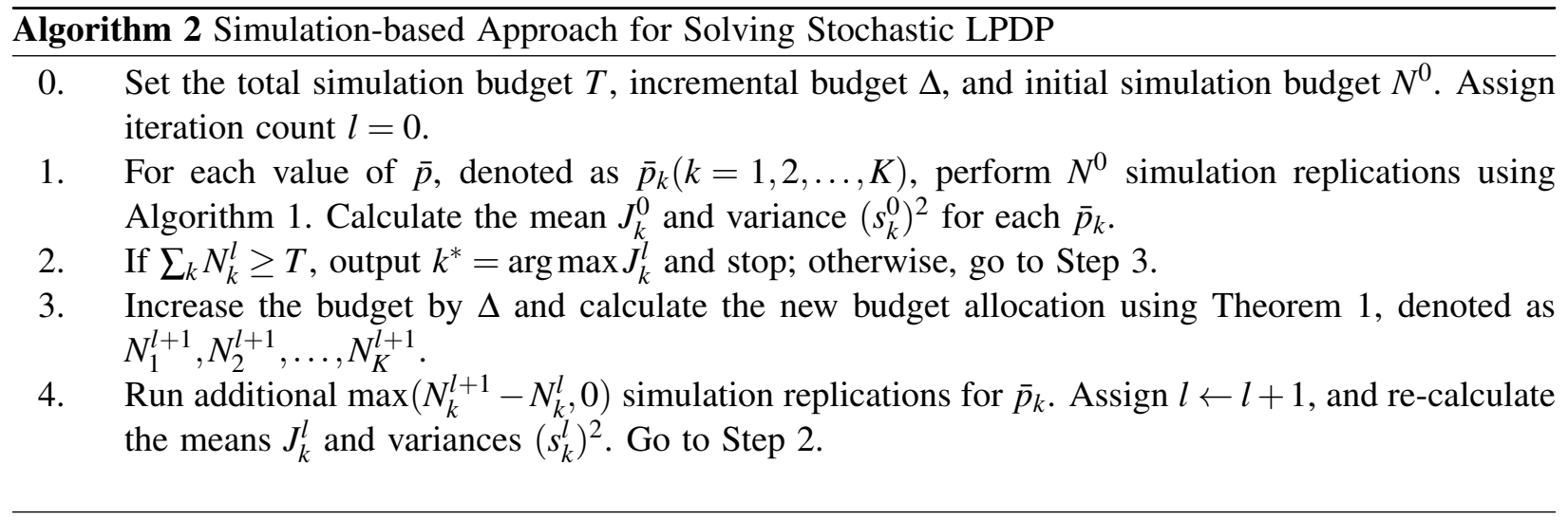

\section{NUMERICAL EXAMPLES}

In this section, we report our computational results on stochastic LPDPs. The experiment settings are as follows.

Map and Locations: We generate locations in a rectangle map. For each location pair, the distance between the two locations is the Euclidean distance on the map.

Deterministic Loads: Generate $X$ loads randomly on the origin-destination location pairs. The handling time of each load is randomly generated as Uniform $(0.25,0.75)$ hour. The earliest starting time for each load is generated uniformly between 7AM and 3PM, and the length of the pickup time window is set to Uniform $(5,10)$ hour. The revenue of serving a load is set to $V \cdot($ loaded movement time + handling time), where $V$ Uniform $(60,100)$ is the rate of revenue per service time unit.

Stochastic Loads: The parameters of stochastic loads are generated similarly to those of deterministic loads. The occurrence probability is set to Uniform $(0.5,1)$. For simplification, the probability is constant in the morning and in the afternoon. The total number of stochastic loads is $Y$.

Drivers: The number of drivers is $Z$. For each driver, the start and home locations are randomly assigned among the locations. Each driver's starting work time is randomly set to Uniform $(7,10) \mathrm{AM}$, and 


\section{Chen, Song, and Shi}

each driver works 10 hours per day. The driving speed of each driver is 40 mile per hour. The cost rate per driver is $\$ 15$ per hour, and the traveling cost per vehicle is $\$ 0.5$ per mile.

We generated 6 testing instances as shown in Table 1. The hybrid nested partitions and mathematical programming (HNP-MP) approach (Pi, Pan, and Shi 2008) is used for solving each deterministic model ((2) - (8), (11)). The dual simplex method is used as the linear programming (LP) solver, and CPLEX is used as the mixed integer programming (MIP) solver.

Table 1: Settings of testing instances.

\begin{tabular}{cccc}
\hline Instance & $\mathrm{X}$ & $\mathrm{Y}$ & $\mathrm{Z}$ \\
\hline 1 & 60 & 10 & 15 \\
2 & 60 & 20 & 20 \\
3 & 80 & 20 & 25 \\
4 & 80 & 30 & 30 \\
5 & 100 & 30 & 40 \\
6 & 100 & 40 & 50 \\
\hline
\end{tabular}

The computational results are summarized in Table 2 . We consider $\bar{p} \in\{0.5,0.6,0.7,0.8,0.9,1\}$. When $\bar{p}=1$, it means no stochastic load is considered in the planning, which is equivalent to applying deterministic LPDP in a dynamic manner. For each instance, the mean profit and standard deviation is presented for each value of $\bar{p}$. It can be observed that for instances $1-4, \bar{p}=0.8$ is the best choice, while $\bar{p}=0.7$ is the best for instance 5 - 6. It indicates that for larger scale of problems, it is more beneficial to consider more stochastic loads. It is also clear that $\bar{p}=1$ is among the worst choices for all instances, indicating that considering stochastic loads in the planning is generally beneficial. Since OCBA tend to allocate more simulation replications to better solutions, the result with a higher mean value usually has a lower variance.

Table 2: Numerical results.

\begin{tabular}{c|cccccc}
\hline \multirow{2}{*}{ Instance } & \multicolumn{5}{|c}{ Mean Profit (Std) for $\bar{p}$} \\
& 0.5 & 0.6 & 0.7 & 0.8 & 0.9 & 1.0 \\
\hline 1 & $2009(97)$ & $2098(87)$ & $2231(84)$ & $2286(78)$ & $2201(94)$ & $2067(90)$ \\
2 & $2167(108)$ & $2299(104)$ & $2471(102)$ & $2556(89)$ & $2401(115)$ & $2218(98)$ \\
3 & $3011(162)$ & $3098(154)$ & $3046(145)$ & $3228(126)$ & $3187(125)$ & $2945(157)$ \\
4 & $3213(183)$ & $3182(168)$ & $3328(142)$ & $3477(119)$ & $3316(159)$ & $3276(171)$ \\
5 & $4145(202)$ & $4194(183)$ & $4312(165)$ & $4276(178)$ & $4078(214)$ & $4109(197)$ \\
6 & $4389(251)$ & $4371(206)$ & $4676(171)$ & $4487(187)$ & $4504(195)$ & $4299(220)$ \\
\hline
\end{tabular}

\section{DISCUSSION AND FUTURE RESEARCH}

In this paper, we extend the deterministic LPDP model into the stochastic LPDP. We first review the mathematical model and solution approaches for the deterministic LPDP. Then, the problem description and mathematical modeling of stochastic LPDP are discussed. When the new objective is to maximize the expected profit, the stochastic problem can be written into a MIP problem with the same mathematical structure as the deterministic version, by assuming the traveling time does not change whether or not a stochastic load materializes. Utilizing this conclusion, we convert the original problem into a simulationbased optimization problem, and propose an efficient algorithm to solve the problem. Our experiments provide the optimal strategy to consider these stochastic loads in the planning process, and confirm the benefits compared to deterministic LPDP.

To extend current research, we plan to implement the idea of considering stochastic loads in real industrial setting. In some cases, businesses would also like to consider a risk-adjusted expected profit, 


\section{Chen, Song, and Shi}

which makes the objective function non-linear. Existing methods are not designed to deal with non-linear problems, and hence research along this line is needed.

\section{REFERENCES}

Arunapuram, S., K. Mathur, and D. Solow. 2003. "Vehicle Routing and Scheduling with Full Truckloads". Transportation Science 37 (2): 170-182.

Berbeglia, G., J.-F. Cordeau, and G. Laporte. 2010. "Dynamic Pickup and Delivery Problems". European Journal of Operational Research 202 (1): 8-15.

Campbell, A. M., and M. Savelsbergh. 2004. "Efficient Insertion Heuristics for Vehicle Routing and Scheduling Problems". Transportation Science 38 (3): 369-378.

Chen, C. H., and L. H. Lee. 2010. Stochastic Simulation Optimization: An Optimal Computing Budget Allocation. World Scientific Publishing Co.

Chen, C. H., J. Lin, E. Yücesan, and S. E. Chick. 2000. "Simulation Budget Allocation for Further Enhancing the Efficiency of Ordinal Optimization". Journal of Discrete Event Dynamic Systems: Theory and Applications 10:251-270.

Erera, A. L., and K. R. Smilowitz. 2008. "Intermodal Drayage Routing and Scheduling". In Intelligent Freight Transportation, edited by P. A. Ioannou, 171-188. CRC Press.

Funke, B., T. Grunert, and S. Irnich. 2005. "Local Search for Vehicle Routing and Scheduling Problems: Review and Conceptual Integration". Journal of Heuristics 11 (4): 267-306.

Ileri, Y. 2006. Drayage Optimization in Truck/Rail Networks. Ph. D. thesis, Georgia Institute of Technology.

Lu, Q., and M. Dessouky. 2004. "An Exact Algorithm for the Multiple Vehicle Pickup and Delivery Problem”. Transportation Science 38 (4): 503-514.

Morlok, E. K., and L. N. Spasovic. 1994. "Redesigning Rail-Truck Intermodel Drayage Operations for Enhanced Service and Cost Performance". Transportation Research Forum 34:16-31.

Pi, L., Y. Pan, and L. Shi. 2008. "Hybrid Nested Partitions and Mathematical Programming Approach and Its Applications". IEEE Transactions on Automation Science and Engineering 4 (5): 573-586.

Powell, W. B., and T. Carvalho. 1998. "Dynamic Control of Logistics Queueing Networks for Large Scale Fleet Management". Transportation Science 32 (2): 90-109.

Powell, W. B., J. Shapiro, and H. P. Simao. 2002. "An Adaptive, Dynamic Programming Algorithm for the Heterogeneous Resource Allocation Problem". Transportation Science 36 (2): 231-249.

Savelsbergh, M. W. P., and M. Sol. 1995. "The General Pickup and Delivery Problem". Transportation Science 29 (1): 17-29.

Wang, X., and A. C. Regan. 2002. "Local Truckload Pickup and Delivery with Hard Time Window Constraints". Transportation Research Part B 36:78-94.

Xu, H., Z.-L. Chen, S. Rajagopal, and S. Arunapuram. 2001. "Solving a Practical Pickup and Delivery Problem". Transportation Science 37 (3): 347-364.

\section{AUTHOR BIOGRAPHIES}

WEIWEI CHEN is a Scientist in the Management Sciences Lab at the General Electric Global Research Center, NY, USA. He received the B.S. and M.S. degree in Automation from Tsinghua University, Beijing, China, in 2003 and 2006, respectively, and the M.S. and Ph.D. degree in Industrial Engineering from the University of Wisconsin-Madison in 2007 and 2010, respectively. He is a member of INFORMS and IEEE. His research interests include large-scale optimization, simulation optimization, and data mining, in the application areas of logistics, energy, aviation, and healthcare. His email address is chenw@ge.com. This paper is his work at the University of Wisconsin-Madison.

JIE SONG is a postdoc in the Department of Industrial Engineering and Management at Peking University, Beijing, China. She received the B.S. degree in Applied Mathematics from Peking University, Beijing, 
China, in 2004, and the M.S. and Ph.D. degree in Industrial Engineering from Tsinghua University in 2007 and 2010, respectively. She is a member of INFORMS and IEEE. Her research interests are simulation optimization, queueing network modeling in the application areas of logistics, healthcare and production. Her email address is songjie@coe.pku.edu.cn.

LEYUAN SHI is a Professor in the Department of Industrial and Systems Engineering at the University of Wisconsin-Madison. She received the B.S. degree in Mathematics from Nanjing Normal University, Nanjing, China, in 1982, the M.S. degree in Applied Mathematics from Tsinghua University, Beijing, China, in 1985, and the M.S. degree in Engineering and the Ph.D. degree in Applied Mathematics from Harvard University in 1990 and 1992, respectively. She is a Fellow of IEEE and a member of INFORMS. Her research is devoted to the theory and applications of large-scale optimization algorithms, discrete-event simulation and modeling, and analysis of discrete dynamic systems. She is currently an associate editor of the IEEE Transactions on Automation Science and Engineering, Discrete Event Dynamic Systems, and the Journal of Methodology and Computing in Applied Probability. Her email address is leyuan@engr.wisc.edu. 